ARTICLE

DOI: $10.1038 / \mathrm{s} 41467-018-04736-1$

\title{
Selective manipulation of electronically excited states through strong light-matter interactions
}

Kati Stranius ${ }^{1}$, Manuel Hertzog ${ }^{1} \&$ Karl Börjesson ${ }^{1}$

Strong coupling between light and matter leads to the spontaneous formation of hybrid light-matter states, having different energies than the uncoupled states. This opens up for new ways of modifying the energy landscape of molecules without changing their atoms or structure. Heavy metal-free organic light emitting diodes (OLED) use reversed intersystem crossing (RISC) to harvest light from excited triplet states. This is a slow process, thus increasing the rate of RISC could potentially enhance OLED performance. Here we demonstrate selective coupling of the excited singlet state of Erythrosine B without perturbing the energy level of a nearby triplet state. The coupling reduces the triplet-singlet energy gap, leading to a four-time enhancement of the triplet decay rate, most likely due to an enhanced rate of RISC. Furthermore, we anticipate that strong coupling can be used to create energyinverted molecular systems having a singlet ground and lowest excited state.

\footnotetext{
${ }^{1}$ Department of Chemistry and Molecular Biology, University of Gothenburg, Kemigården 4, 41296 Gothenburg, Sweden. Correspondence and requests for materials should be addressed to K.Bör. (email: karl.borjesson@gu.se)
} 
C hemists can today synthesize virtually any molecule imaginable. By fine-tuning the molecular structure, optimized physical, chemical, or biological properties are routinely achieved. However, even though molecular optimization has reshaped the world we live in, there is always a point at which the laws of physics limits the performance of molecular systems. Tailoring of molecular properties can be achieved through strong coupling between molecular states and the zeropoint fluctuations of the electromagnetic field (vacuum field $)^{1-4}$. The formed hybrid states (exciton polaritons) have unique chemical and physical properties and can be viewed as a linear combination of light (vacuum field) and matter (molecules). Strong coupling between organic molecules and light has gathered considerable attention in the past couple of years due to the vast number of possible applications it offers in physical and chemical sciences ${ }^{5}$. For example, strong coupling has been observed in molecular crystals ${ }^{6-8}$, J-aggregates ${ }^{9-13}$, polymers ${ }^{14-}$ 16 , small molecules in a polymer or silicon dioxide matrix ${ }^{17-26}$, large light-harvesting complexes $^{27}$ and even liquid crystals ${ }^{28}$ '. Furthermore, it has been shown to change chemical reactivity ${ }^{29}$, work function ${ }^{23}$, and phase transitions ${ }^{30}$; increase electronic conductivity $^{31}$ and energy transfer, and modify ground-state thermodynamics $^{22}$ and reaction-energy landscapes ${ }^{20}$. Strong coupling between single molecules and a vacuum field has now also been realized ${ }^{32}$.

Optical cavities, e.g., two parallel mirrors, can be used to couple light and matter. The coupling leads to the formation of two new optically allowed hybrid light-matter states, $P^{-}$and $P^{+}$, which are separated in energy by the Rabi splitting ( $\hbar \Omega_{\mathrm{R}}$; Fig. 1a). Using the rotating wave approximation, in the limit of large number of molecules $(N)$, the Hamiltonian of the system can be approximated by ${ }^{2}$ :

$$
\begin{gathered}
\hat{\mathcal{H}}=\hat{\mathcal{H}}_{\text {mol }}+\hat{\mathcal{H}}_{\mathrm{cav}}+\hat{\mathcal{H}}_{\mathrm{int}} \approx \hbar \omega_{0}\left(-\frac{N}{2}+\hat{b}^{\dagger} \hat{b}\right) \\
+\hbar \omega \hat{a}^{\dagger} \hat{a}+\hbar g \sqrt{N}\left(\hat{a}^{\dagger} \hat{b}+\text { h.c. }\right)
\end{gathered}
$$

where, $g=-\mathbf{d} \mathcal{E} / \hbar$ is the coupling constant between the cavity mode and the electronic transition of the molecule, $\mathbf{d}$ is the respective transition dipole moment, and $\varepsilon$ is the vacuum electric field. It should be noted that $\varepsilon$ is finite even in the absence of an external electromagnetic field. This is due to vacuum fluctuations inside the cavity and all measurements and considerations here is made in this low photon regime. Solving the eigenmodes of Eq. 1 gives the magnitude of the vacuum Rabi splitting:

$$
\hbar \Omega_{\mathrm{R}}=2 g \sqrt{N}
$$

The magnitude of the Rabi splitting is thus proportional to the transition dipole moment associated with the state being hybridized and the square root of the concentration of the molecule inside the cavity.

The linear dependence of the Rabi splitting on the magnitude of the transition dipole moment (Eq. 2) has profound practical and theoretical implications. The electronic ground state of an organic molecule is of singlet character. Furthermore, Hund's rule states that triplet states always are lower in energy than their corresponding singlet states. Thus the first excited state of an organic molecule is a triplet state (dark state), having a low transition dipole moment to the ground state. The inefficient emission of light from triplet states is problematic in the field of light-emitting organic electronics. To address this, there has been a considerable activity in the research field of thermally activated delayed fluorescence, in which molecules are engineered to have as small a triplet-singlet energy difference as possible, enabling thermal excitation of the singlet state from triplet states ${ }^{33}$. This concept has been relatively successful, but still, the rate of emission from a singlet state is several orders of magnitude faster compared to the rate of delayed fluorescence ${ }^{34}$. However, the dependence of the Rabi splitting on the magnitude of the transition dipole moment suggests that strong coupling could be used to selectively modify the energy levels of singlet states, without significantly perturbing the energy levels of triplet states. This would enable decreasing the energy difference between hybrid states of singlet character and triplet states, possibly leading to an enhanced rate of thermally activated reversed intersystem crossing (RISC) and potentially enabling the use of conventional fluorophores (e.g., fluorescein and rhodamine) in efficient organic light-emitting devices.

Here we demonstrate on the possibility to selectively tune excited state processes by modifying the energy landscape through coupling with the vacuum field. We start by showing that it is possible to couple a singlet state to the vacuum field without perturbing the energy level of a nearby triplet (dark) state. Using time-resolved spectroscopy, we continue with showing that the triplet decay rate is four times increased in the strong coupling regime, most likely due to an enhanced rate of RISC. Finally, by observing the temperature dependence of the triplet decay rate, the lowering of the activation energy of RISC is in good agreement with the energy difference between the excitonic and lower polaritonic state, as measured with absorbance spectroscopy.

\section{Results}

Formation of hybrid light-matter states of Erythrosine B. We chose Erythrosine B (ErB) as a model compound for observing

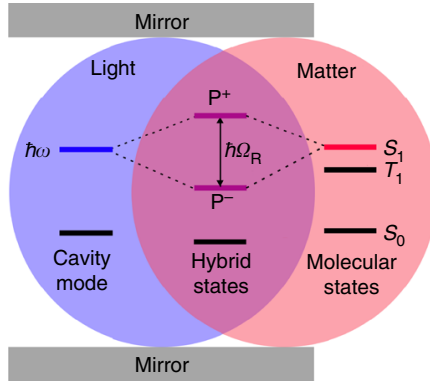

b

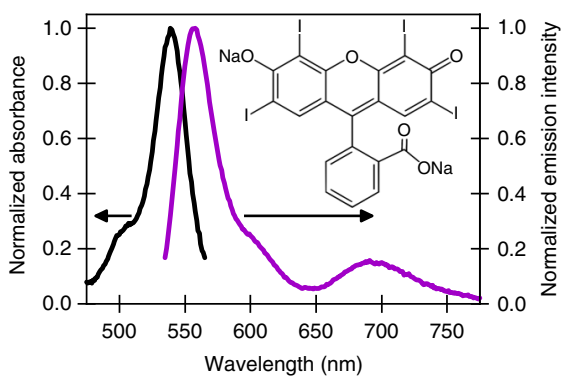

C

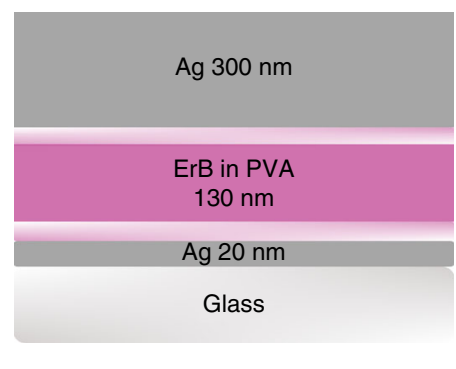

Fig. 1 Molecular system for realizing strong light-matter coupling. a Jablonski diagram presenting strong coupling of a molecular state $\left(S_{1}\right)$ with a cavity mode $(\hbar \omega)$ that has the same energy and leads to the formation of two new hybrid light-matter states, $P^{+}$and $P^{-}$, which are separated in energy by the Rabi splitting $\left(\hbar \Omega_{R}\right)$. b Absorption (black line) and emission (purple line) spectra of $1 \mathrm{wt} \%$ ErB in a PVA matrix deposited on a glass substrate. Inset shows the chemical structure of ErB. c Structure of the non-transparent Fabry-Pérot cavity, where two Ag mirrors sandwich a PVA film containing ErB. PMMA 10 $\mathrm{nm}$ films, deposited between the mirror and chromophore layer, were used to prevent direct contact between ErB and the Ag mirror 

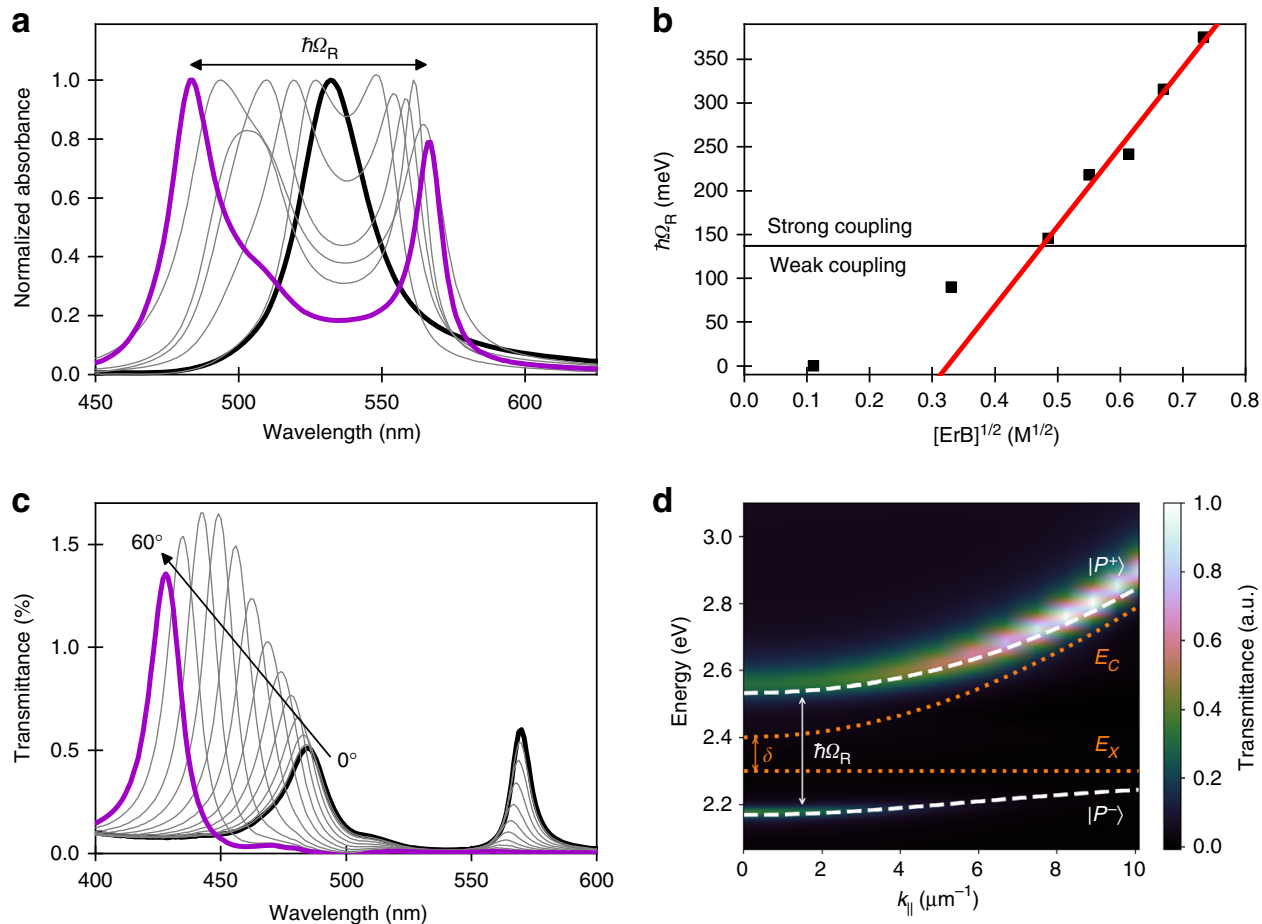

Fig. 2 Characterization of hybrid light-matter states. a Absorbance spectra of ErB/PVA films inside a cavity that has an ErB concentration between $0.01 \mathrm{M}$ (black) and $0.54 \mathrm{M}$ (purple). The thickness of the cavities were optimized as to give a roughly equal magnitude of the lower and upper polariton absorption. $\mathbf{b}$ The linear dependence of the Rabi splitting in the strong coupling regime on the square root of [ErB]. The strong/weak coupling limit is visualized as a line at $140 \mathrm{meV}$. c Transmission spectra of a $0.54 \mathrm{M} \mathrm{ErB/PVA} \mathrm{film} \mathrm{inside} \mathrm{a} \mathrm{cavity} \mathrm{having} \mathrm{two} \mathrm{semitransparent} \mathrm{mirrors} \mathrm{as} \mathrm{a} \mathrm{function} \mathrm{of}$ incidence angle. $\mathbf{d}$ Dispersion plot constructed from the data in (c) (color map) overlaid with fitted polariton dispersion (white dashed line). The uncoupled non-dispersive ErB absorption $\left(E_{x}\right)$ and cavity dispersion $\left(E_{c}\right)$ are shown as dashed lines. The Rabi splitting $\left(\hbar \Omega_{R}\right)$ is the energy gap between $P^{-}$and $P^{+}$at the intersection (or minimum gap) of $E_{\mathrm{x}}$ and $E_{\mathrm{c}}$

the dynamics of excited singlet and triplet states. ErB is the tetraiodized derivative of fluorescein (Fig. 1b) and when dissolved in a rigid polymer matrix, it simultaneously exhibits both fluorescence and phosphorescence (Fig. 1b). The absorbance spectrum shows, apart from a small shoulder, a single transition centered at $538 \mathrm{~nm}$, with an oscillator strength of $0.5^{35}$. The fluorescence is centered at $558 \mathrm{~nm}$ and is a mirror image to the absorbance, and the phosphorescence is relatively broad and structureless with a maximum at $691 \mathrm{~nm}$. Thus information on the energy levels of the lowest excited singlet and triplet states are realized in a single experiment at room temperature. Furthermore, ErB exhibits e-type delayed fluorescence $^{36}$, i.e., fluorescence arising from thermal population of the singlet state from a triplet state, which enables straightforward monitoring of singlet/triplet dynamics through time-resolved spectroscopy.

To probe light-matter interactions, ErB was inserted into a Fabry-Pérot cavity (Fig. 1c). The strong coupling regime is characterized by the cavity mode splitting into two new bands, $P^{-}$ and $P^{+}$, separated by the Rabi splitting (Fig. 2a). Strong coupling is achieved when the magnitude of the Rabi splitting is larger than the full width at half maximum (FWHM) of both the ErB absorbance band and the cavity mode, which occurred when the Rabi splitting was $>140 \mathrm{meV}$. Entrance to the strong coupling regime can be also seen in Fig. $2 b$ where the linear dependence of the Rabi splitting on the square root of $[\mathrm{ErB}]$ is seen in the strong coupling regime (Eq. 2). The hybrid states are dispersive by nature, and thus they have properties that are different from those of the individual molecular states or cavity modes (Fig. 2c, d). With an increasing angle of incidence, the absorbance spectra show a hypsochromic shift together with a reduction of $P^{-}$and increment of $P^{+}$absorption. Transfer matrix calculations confirmed the experimental observations (Supplementary Fig. 1). From the Hopfield coefficients that quantify the exciton and photon ratio in each of the polariton branches (Supplementary Fig. 2), we observed that, at normal incidence angle, both the molecular and optical modes contribute roughly equally to the hybrid states. However, at larger $k_{\|}$values, $P^{+}$has increasing photonic contribution following the cavity dispersion (Eq. 7 in Methods) and $\mathrm{P}^{-}$has increasing material contribution following the excitonic transition energy. Thus all optical measurements from here on were recorded normal to the cavity surface.

Lowering of the triplet-singlet energy gap. By making use of the optimal conditions ascertained above, we were able to set up and study the difference in energy between the triplet and hybrid singlet states. The cavity containing $0.54 \mathrm{M}$ ErB displays the largest Rabi splitting ( $375 \mathrm{meV}$; Fig. 2a), which would correspond to a lowering of the $P^{-}$- state energy by $187.5 \mathrm{meV}$ compared to the uncoupled singlet-state energy. However, owing to asymmetry of the ErB absorbance (Fig. 1b), the splitting was not symmetric and the lower polaritonic band $P^{-}$was actually shifted by 135 meV compared to the uncoupled state. The lowering of the $P$ - state energy can also be followed by the emission arising from the $P^{-}$state (Fig. 3a). Fluorescence arising from uncoupled ErB has a maximum at $543 \mathrm{~nm}$ (Supplementary Table 1). As the strong coupling regime is reached through an increase in $\mathrm{ErB}$ concentration, the $P^{-}$emission is gradually red-shifted. The emission maximum of the cavity containing $0.54 \mathrm{M}$ of $\operatorname{ErB}$ is shifted by $24 \mathrm{~nm}(96 \mathrm{meV})$ compared to uncoupled ErB fluorescence (Supplementary Table 1, Supplementary Note 1). In 

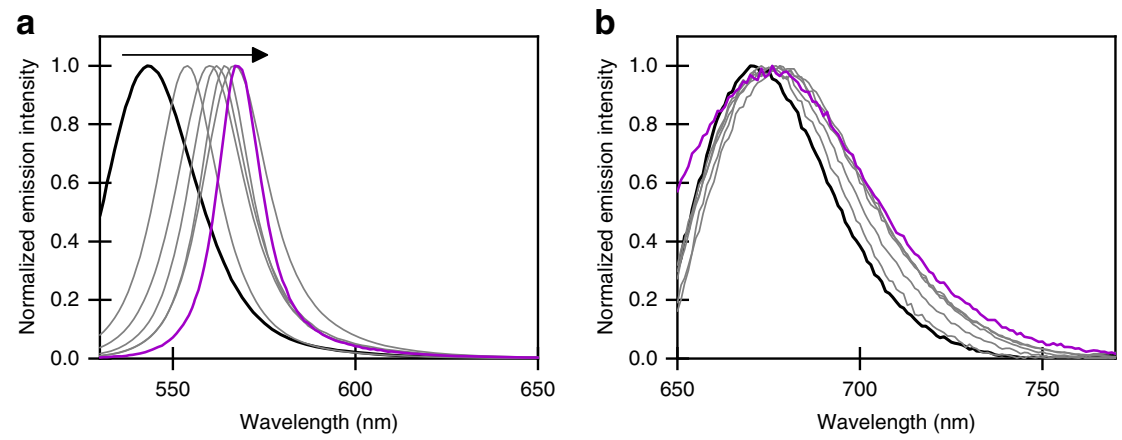

Fig. 3 Selective coupling of excited states and decrease of triplet-singlet energy gap. a Fluorescence/ $P^{-}$emission and $\mathbf{b}$ phosphorescence spectra of ErB/ PVA films inside a cavity with an ErB concentration between 0.01 (black) and $0.54 \mathrm{M}$ (purple), excited at the maximum of the $P^{+}$absorbance. The clean phosphorescence band was obtained by subtracting the fluorescence tail from the phosphorescence spectra (Supplementary Fig. 3)

a

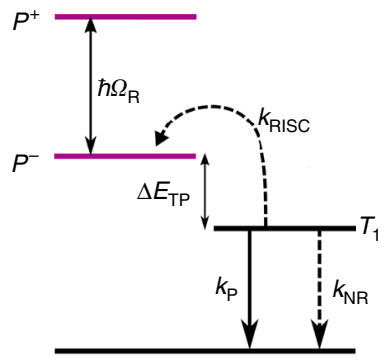

C

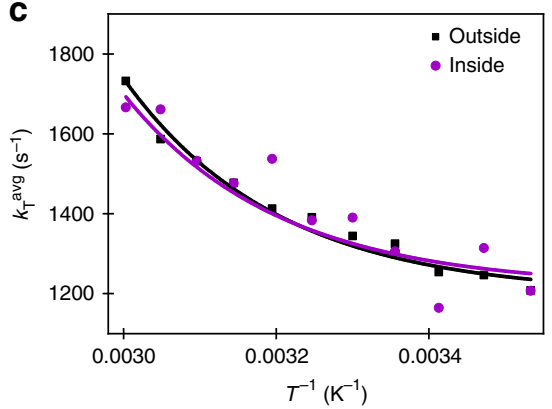

b

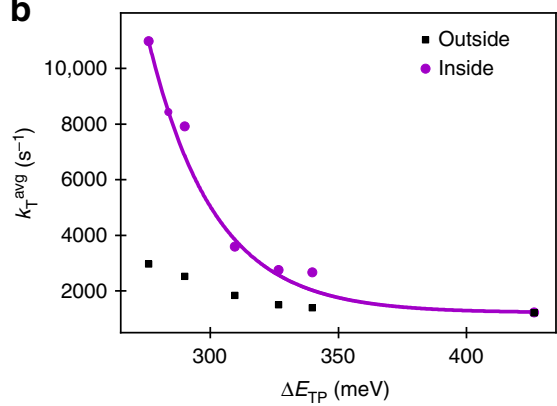

d

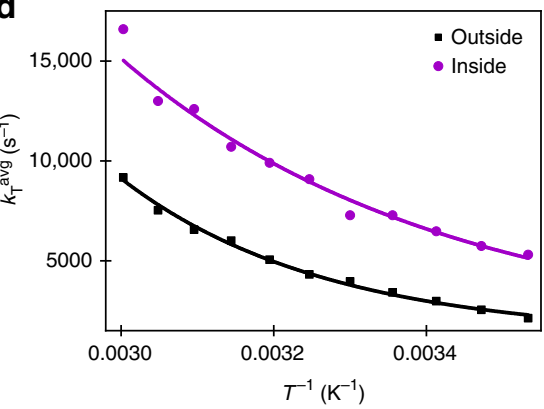

Fig. 4 Increased rate of reversed intersystem crossing. a The energy diagram describing the kinetics of the triplet-state depopulation pathways inside a cavity. $k_{\mathrm{P}}, k_{\mathrm{NR}}$, and $k_{\mathrm{RISC}}$ are the rates of phosphorescence, non-radiative decay, and reverse intersystem crossing, respectively. $\Delta E_{T P}$ is the energy difference between $T_{1}$ and $P^{-}$. b The increase of the average total and the fitted rate constant (Eq. 3) of the triplet-state depopulation $\left.\left(k^{\text {avg }}\right)_{T}\right)$ inside the cavity as a function of the energy difference between $T_{1}$ and $P^{-}\left(\Delta E_{\mathrm{TP}}\right)$. The rate constants outside the cavity with equivalent concentration are shown for comparison. c, $\mathbf{d}$ Temperature dependence of the average total rate constant of the triplet-state depopulation $\left(k^{\text {avg }}\right.$ ) and exponential fit (Eq. 3 ) for $0.02 \mathrm{M}$ (c) and $0.54 \mathrm{M}$ (d) ErB/PVA films outside and inside a cavity. Samples were excited at the maximum of the $P^{+}$absorbance peak and monitored at 690 nm

contrast, the phosphorescence band inside the cavity was not redshifted compared to reference samples when the concentration of ErB was increased (Fig. 3b, Supplementary Table 1 and Supplementary Fig. 3). The lack of concentration dependence of phosphorescence shows that it is possible to couple the singlet state to the vacuum field without significantly perturbing the corresponding triplet states. Thus, with an increase in the coupling strength of the singlet (polaritonic) state, a simultaneous reduction of the energy difference between the triplet and singlet (polaritonic) state $\left(\Delta E_{\mathrm{TS}}\right)$ is achieved.

To show that emission occurs as a consequence of excitation to the polaritonic states, rather than to uncoupled molecules, excitation spectra of cavities with different ErB concentrations were monitored at both the lower polariton and the phosphorescence emission maxima (Supplementary Fig. 4). The excitation spectra are in reasonable agreement (Supplementary Note 2) with corresponding absorbance spectra for all cavities at both emission wavelengths. Thus relaxation from the upper and lower polariton states to the triplet state is equally efficient. In addition, the presence of polaritonic peaks in the excitation spectra monitored at the phosphorescence maxima proofs that the polaritons (or excitation reservoir) can undergo ISC as reported earlier ${ }^{37}$. Furthermore, no emission can be seen when exciting at wavelengths where uncoupled ErB absorb light.

Since the triplet-state energy is unaffected in the cavity, we assumed that the triplet-singlet (polaritonic) energy gap $\left(\Delta E_{\mathrm{TP}}\right)$ decreases with the Rabi splitting. At low concentration, $\Delta E_{\mathrm{TS}}$ inside the cavity was $436 \mathrm{meV}$ and it was determined from the spectral separation of the maxima of the fluorescence and phosphorescence (Supplementary Table 1). The obtained value agrees well with the previously reported value for $\operatorname{ErB}$ in a polyvinyl alcohol (PVA) matrix (Supplementary Note 3) ${ }^{36}$. Figure $4 \mathrm{a}$ represents the energy diagram that describes the kinetics of the triplet-state depopulation pathways after the triplet 
state is populated by ISC from $S_{1} / P^{-}$to $T_{1}$. The triplet state can be assumed to be populated instantly since ISC from the initially excited $S_{1} / P^{-}$state to $T_{1}$ is much faster (nanoseconds) than the deactivation rates (microseconds) of $T_{1}$. The phosphorescence lifetime is given by:

$\tau_{\mathrm{T}}^{-1}=k_{\mathrm{T}}=k_{\mathrm{P}}+k_{\mathrm{NR}}+k_{\mathrm{RISC}}=k_{\mathrm{P}}+k_{\mathrm{NR}}+k_{\mathrm{ISC}} \exp \left(-\frac{\Delta E_{\mathrm{TS} / \mathrm{P}}}{R T}\right)$

where $k_{\mathrm{T}}$ is the total rate constant of the triplet state, $k_{\mathrm{P}}$ is the radiative rate of phosphorescence, $k_{\mathrm{NR}}$ is the non-radiative decay rate, and $k_{\mathrm{RISC}}$ and $k_{\mathrm{ISC}}$ are the rates of RISC from $T_{1}$ to $S_{1} / P^{-}$ and ISC from $S_{1} / P^{-}$to $T_{1}$, respectively. The probability that the molecule will overcome the energy barrier between the triplet state and the first excited singlet/polaritonic state is given by the Boltzmann distribution factor, $\exp \left(-\Delta E_{\mathrm{TS} / \mathrm{P}} / R T\right)^{36}$.

Increased RISC due to strong coupling. To determine how the lowering of $\Delta E_{\mathrm{TP}}$ affects the excited state dynamics of ErB, timeresolved phosphorescence decays were measured. The triplet state was monitored since it does not show any coupling to the vacuum field and thus remains stationary in energy in the strong coupling regime. The phosphorescence lifetimes $\left(\tau_{\mathrm{T}}\right)$ were obtained from the measured phosphorescence decays by using stretchedexponential tail-fits (Supplementary Note 4). The phosphorescence decays and fitting results for all uncoupled and coupled samples are shown in Supplementary Fig. 5 and Supplementary Table 2, respectively. Figure $4 \mathrm{~b}$ shows the average total rate constant of the triplet state $\left(k^{\mathrm{avg}} \mathrm{T}\right)$ depopulation as the energy difference between $T_{1}$ and $P^{-}\left(\Delta E_{\mathrm{TP}}\right)$ is decreasing (Supplementary Note 5, Supplementary Fig. 6). At low concentrations, when the system is not in the strong coupling regime, the rate of triplet decay is the same inside the cavity as compared to a bare film, thus indicating that the presence of the cavity does not induce any simple electromagnetic effects (such as the Purcell effect). However, at high concentrations of $\mathrm{ErB}$ (thus in the strong coupling regime), the phosphorescence decay was four times less inside the cavity as compared to outside. Furthermore, the change of $k_{\mathrm{T}}$ can successfully be reproduced by Eq. 3 (Supplementary Table 3), indicating that the change in $k_{\mathrm{T}}$ is due to a reduced triplet-polaritonic energy gap. Importantly, the photophysical pathways of triplet-state deactivation are modified even though only the singlet state was coupled to the vacuum field.

To further strengthen the hypothesis that the increase in the rate constant of triplet-state deactivation is due to a lowering of $\Delta E_{\mathrm{TP}}$, the temperature dependence of the phosphorescence lifetime for uncoupled $(0.01 \mathrm{M})$ and strongly coupled $(0.54 \mathrm{M})$ ErB was determined (Fig. 4c, d, respectively; see Supplementary Fig. 7 and Supplementary Table 5-6 for raw data). The temperature dependence of the phosphorescence rate constants of uncoupled $\operatorname{ErB}(0.01 \mathrm{M})$ inside the cavity overlaps well with the values outside a resonant cavity (Fig. 4c). However, the observed temperature dependence of the phosphorescence rate constants of ErB at high concentration $(0.54 \mathrm{M})$ inside the cavity (strongly coupled) differs from those outside a resonant cavity (Fig. 4d). To estimate the change of the triplet-singlet/polaritonic energy gap inside the cavity, Eq. 3 was used to fit the temperature dependence of the phosphorescence (Supplementary Table 6). The obtained $\Delta E_{\mathrm{TS} / \mathrm{P}}$ values were $322 \mathrm{meV}$ and $204 \mathrm{meV}$ for $\mathrm{ErB}$ at $0.54 \mathrm{M}$ outside and inside the cavity, respectively. This corresponds to a lowering of the triplet-singlet/polaritonic energy gap by $118 \mathrm{meV}$. Although the $\Delta E_{\mathrm{TS} / \mathrm{P}}$ values are slightly smaller than the values obtained from steady-state analysis, the lowering of the energy gap agrees well with the shift in the lower polaritonic absorption band compared to the uncoupled state (Supplementary Table 7). This supports the hypothesis that the decrease of the phosphorescence lifetime is due to an increased rate of RISC caused by a lowering of the triplet-singlet/ polaritonic energy gap. Interestingly, in the temperaturedependence analysis, a reduction of the fitted $k_{\text {ISC }}$ was noticed. Thus the coupling between the triplet and polaritonic states seems to be slightly lower as compared to the coupling between the triplet and singlet states. The lower coupling works against the lowering of $\Delta E_{\mathrm{TS} / \mathrm{P}}$ and the effect is thus unfavorable in singletharvesting applications. However, it is too early to say if a lower coupling between triplet and polaritonic states is system specific or a general feature for organic molecules in the strong coupling regime.

\section{Discussion}

We have successfully coupled the singlet excited state of ErB to the vacuum field, without perturbing the energy of the corresponding triplet state. By doing so, the triplet-singlet/polaritonic energy gap was reduced, resulting in an increased triplet decay rate, presumably due to an increased rate of RISC. We envisage that the described concept could be used to lower the energy of excited singlet states under that of the corresponding triplet states. This would allow for molecular systems to have a ground and first excited state of singlet character and lead to numerous practical applications in applied fields, such as light-emitting organic electronics.

\section{Methods}

Cavity preparation. The Fabry-Pérot cavities were built on glass substrates $(25 \times$ $25 \mathrm{~mm}^{2}$ ), which were precleaned by sonication for $15 \mathrm{~min}$ in alkaline solution ( $0.5 \%$ of Hellmanex in distilled water), and then rinsed with water and sonicated for $1 \mathrm{~h}$ in water and ethanol, respectively. The cleaned glass substrates were dried in an oven overnight before cavity preparation. Polymethyl methacrylate (PMMA, Sigma Aldrich) was dissolved in toluene $\left(5 \mathrm{mg} \mathrm{mL}^{-1}\right)$. ErB (Sigma Aldrich) was dissolved in water containing PVA (88\% hydrolyzed, Acros Organics, 25-35 mg $\mathrm{mL}^{-1}$ ). Polymer films were deposited by spin-coating (Laurell Technologies WS650), at speeds optimized to give roughly $10 \mathrm{~nm}$ PMMA and $130 \mathrm{~nm}$ PVA/ErB films. Thickness of the polymer films were measured using profilometry (KLA Tencor D-100). Ag mirrors were fabricated by vacuum sputtering deposition (HEX Korvus Technologies). A semitransparent $20 \mathrm{~nm} \mathrm{Ag} \mathrm{film} \mathrm{was} \mathrm{sputtered} \mathrm{on} \mathrm{top} \mathrm{of}$ the glass plate and the thick $300 \mathrm{~nm}$ films was sputtered on top of the polymer layers to form a sealed cavity that could be stored in nitrogen atmosphere for months without there being any effects of Ag oxidation on the cavity properties. PMMA $10 \mathrm{~nm}$ films were used to prevent direct contact between $\mathrm{ErB}$ and the $\mathrm{Ag}$ mirrors (direct contact could affect both radiative and non-radiative rate constants of ErB). The thickness of the PVA/ErB film was optimized to give the $\lambda / 2$ cavity mode in resonance with the absorbance maximum of ErB at $538 \mathrm{~nm}$.

To determine the absorbance of the cavity, we used one semitransparent ( 20 $\mathrm{nm})$ and one non-transparent $(300 \mathrm{~nm})$ mirror. The absorbance was calculated ( $A=\log \frac{1}{\mathrm{R}}$ ) by assuming that transmission and scattering by the cavity is zero and probed by using reflectance spectroscopy. The total concentration of ErB inside the cavity was calculated from the absorbance spectra of reference samples with only one Ag mirror. The quality factor $\left(Q=\lambda_{\mathrm{r}} / \Delta \lambda\right)$ of empty cavities was around 15 , which is in line with the FWHM of the absorbance of ErB. For dispersion measurements, transparent cavities were prepared so that both mirrors were $50 \mathrm{~nm}$ thick. Reference samples were prepared using same parameters and solutions for spin-coating the polymer layers and sputtering $300 \mathrm{~nm} \mathrm{Ag} \mathrm{layer} \mathrm{on} \mathrm{top.}$

In addition, because of the low intensity of the phosphorescence from the cavity the lifetime measurements were done for cavities with slightly thinner Ag mirror (18 nm, $Q=18)$, giving a substantial higher signal-to-noise ratio. The absorbance and emission data for these cavities are presented in Supplementary Fig. 8-9.

Optical measurements. Steady-state transmission and reflectance spectra were measured using a spectrophotometer equipped with a small-angle specular reflectance accessory (Lambda 650, Perkin Elmer). Reflectance spectra were measured relative to a standard reflectance mirror and the angular transmittance was measured using a horizontal Glan-Taylor polarizer. Steady-state emission spectra were measured with a spectrofluorometer (Spex Fluorolog, JY Horiba or FLS1000, Edinburgh Instruments). The optical properties of the nontransparent cavities were studied through the glass in front-face geometry.

Direct time-resolved emission decay data were recorded using a nanosecond Nd:YAG Surlite pulsed laser with a repetition frequency of $10 \mathrm{~Hz}$ and an FWHM 
pulse of $7 \mathrm{~ns}$. The desired excitation wavelength was obtained using a Surlite OPO (Continuum). Phosphorescence was detected using a five-stage photomultiplier (Applied Photophysics) and recorded with an oscilloscope interfaced with a custom-made LabVIEW program. The decays were recorded using excitation pulse energy $<0.2 \mathrm{~mJ} \mathrm{~cm}^{-2}$. During the temperature-dependent measurements, the excitation spot was changed a few times to avoid degradation of the sample throughout the duration of the experiments.

Stretched exponential fitting. Phosphorescence decays were analyzed using a stretched exponential function ${ }^{38}$ :

$$
I(t)=I(0) \exp \left[-(t / \tau)^{\beta}\right]
$$

where $I(0)$ is the initial amplitude, $\tau$ is the time constant characterizing the position, and $\beta$ is width of the distribution of decay times. $\beta$ varies from 0 to 1 and quantifies the non-exponential nature of the decay.

In order to compare the distribution of phosphorescence lifetimes, the average phosphorescence time constants were calculated ${ }^{38}$ :

$$
\tau^{\text {avg }}=\tau \Gamma\left(1+\frac{1}{\beta}\right)
$$

where $\Gamma$ is the Gamma function.

Simulating the angle-resolved reflectivity measurements. To model the angleresolved reflectivity measurements, we modeled the system as a coupled oscillator. Inside a Fabry-Pérot cavity, we can model the coupling between an exciton and a photon using the following Hamiltonian ${ }^{39,40}$ :

$$
\hat{H}\left(k_{\|}\right)=\left(\begin{array}{cc}
E_{\mathrm{X}}\left(k_{\|}\right)-i \hbar \Gamma_{\mathrm{X}} & V_{\mathrm{A}} \\
V_{\mathrm{A}} & E_{\mathrm{C}}\left(k_{\|}\right)-i \hbar \Gamma_{\mathrm{C}}
\end{array}\right)
$$

where $E_{\mathrm{X}}$ is the excitonic transition energy, and $\hbar \Gamma_{\mathrm{X}}$ and $\hbar \Gamma_{\mathrm{C}}$ are the broadening of the excitonic transition and the cavity (FWHM). $E_{\mathrm{C}}$ is the energy dispersion of the cavity given by ${ }^{39,40}$.

$$
E_{\mathrm{C}}(\theta)=\frac{E_{0}}{\sqrt{1-\left(\frac{\sin (\theta)}{n_{\text {eff }}}\right)^{2}}}
$$

where $E_{0}=E_{\mathrm{X}}-\delta$ with $\delta$ as the cavity detuning $(\delta=100 \mathrm{meV}), n_{\mathrm{eff}}$ is the refractive index inside the cavity (PVA matrix, $n_{\mathrm{eff}}=1.49$ ), and $\theta$ is the angle of incidence. $V_{\mathrm{A}}$ is the interaction potential representing the coupling of the two oscillators related to the Rabi splitting $\hbar \Omega_{\mathrm{R}}$, when $E_{\mathrm{C}}=E_{X}$ and is given by:

$$
V_{\mathrm{A}}=\frac{1}{2} \sqrt{\left(\hbar \Omega_{\mathrm{R}}\right)^{2}+\left(\hbar \Gamma_{\mathrm{C}}-\hbar \Gamma_{\mathrm{X}}\right)^{2}}
$$

Eigenvalues of $\hat{H}$ give the energy of the upper and lower polariton branch $\left(P^{+}\right.$ and $P^{-}$respectively) with $\left|P^{+}\right\rangle$and $\mid P^{-}>$as eigenvectors:

$$
E^{P^{+} / P^{-}}=\frac{1}{2}\left[E_{\mathrm{X}}+E_{\mathrm{C}}-i\left(\hbar \Gamma_{\mathrm{X}}-\hbar \Gamma_{\mathrm{C}}\right)\right] \pm \sqrt{V_{A}^{2}+\frac{1}{4}\left(E_{\mathrm{X}}-E_{\mathrm{C}}-i\left(\hbar \Gamma_{\mathrm{C}}-\hbar \Gamma_{\mathrm{X}}\right)\right)^{2}}
$$

Data availability. Data are available on request from the authors.

Received: 13 March 2018 Accepted: 18 May 2018

Published online: 11 June 2018

\section{References}

1. Haroche, S. \& Kleppner, D. Cavity quantum electrodynamics. Phys. Today $\mathbf{4 2}$ 24-30 (1989)

2. Törmä, P. \& Barnes, W. L. Strong coupling between surface plasmon polaritons and emitters: a review. Rep. Prog. Phys. 78, 013901 (2014).

3. Ebbesen, T. W. Hybrid light-matter states in a molecular and material science perspective. Acc. Chem. Res. 49, 2403-2412 (2016).

4. Ruggenthaler, M., Tancogne-Dejean, N., Flick, J., Appel, H. \& Rubio, A. From a quantum-electrodynamical light-matter description to novel spectroscopies. Nat. Rev. Chem. 2, 0118 (2018).

5. Martínez-Martínez, L. A., Du, M. F., Ribeiro, R., Kéna-Cohen, S. \& YuenZhou, J. Polariton-assisted singlet fission in acene aggregates. J. Phys. Chem. Lett. 9, 1951-1957 (2018).
6. Holmes, R. J. \& Forrest, S. R. Strong exciton-photon coupling and exciton hybridization in a thermally evaporated polycrystalline film of an organic small molecule. Phys. Rev. Lett. 93, 186404 (2004).

7. Kèna-Cohen, S. \& Forrest, S. R. Room-temperature polariton lasing in an organic single-crystal microcavity. Nat. Photonics 4, 371-375 (2010).

8. Chervy, T. et al. High-efficiency second-harmonic generation from hybrid light-matter states. Nano Lett. 16, 7352-7356 (2016).

9. Schouwink, P., Berlepsch, Hv, Dähne, L. \& Mahrt, R. F. Dependence of Rabi-splitting on the spatial position of the optically active layer in organic microcavities in the strong coupling regime. Chem. Phys. 285, 113-120 (2002).

10. Coles, D. M. et al. Vibrationally assisted polariton-relaxation processes in strongly coupled organic-semiconductor microcavities. Adv. Funct. Mater. 21 , 3691-3696 (2011)

11. Schwartz, T. et al. Polariton dynamics under strong light-molecule coupling. Chemphyschem 14, 125-131 (2012).

12. Coles, D. M., Grant, R. T., Lidzey, D. G., Clark, C. \& Lagoudakis, P. G. Imaging the polariton relaxation bottleneck in strongly coupled organic semiconductor microcavities. Phys. Rev. B 88, 121303 (2013).

13. Wang, S. et al. Quantum yield of polariton emission from hybrid light-matter states. J. Phys. Chem. Lett. 5, 1433-1439 (2014).

14. Takada, N., Kamata, T. \& Bradley, D. D. C. Polariton emission from polysilane-based organic microcavities. Appl. Phys. Lett. 82, 1812-1814 (2003).

15. Plumhof, J. D., Stöferle, T., Mai, L., Scherf, U. \& Mahrt, R. F. Roomtemperature Bose-Einstein condensation of cavity exciton-polaritons in a polymer. Nat. Mater. 13, 247-252 (2014)

16. Zhong, X. et al. Energy transfer between spatially separated entangled molecules. Angew. Chem. Int. Ed. 56, 9034-9038 (2017).

17. Lidzey, D. G. et al. Strong exciton-photon coupling in an organic semiconductor microcavity. Nature 395, 53-55 (1998).

18. Schwartz, T., Hutchison, J. A., Genet, C. \& Ebbesen, T. W. Reversible switching of ultrastrong light-molecule coupling. Phys. Rev. Lett. 106, 196405 (2011).

19. Berrier, A. et al. Active control of the strong coupling regime between porphyrin excitons and surface plasmon polaritons. ACS Nano 5, 6226-6232 (2011).

20. Hutchison, J. A., Schwartz, T., Genet, C., \& Devaux, E. \& Ebbesen, T. W. Modifying chemical landscapes by coupling to vacuum fields. Angew. Chem. Int. Ed. 51, 1592-1596 (2012)

21. Hayashi, S., Ishigaki, Y. \& Fujii, M. Plasmonic effects on strong excitonphoton coupling in metal-insulator-metal microcavities. Phys. Rev. B 86, 045408 (2012)

22. Canaguier-Durand, A. et al. Thermodynamics of molecules strongly coupled to the vacuum field. Angew. Chem. Int. Ed. 52, 10533-10536 (2013).

23. Hutchison, J. A. et al. Tuning the work-function via strong coupling. $A d v$. Mater. 25, 2481-2485 (2013)

24. Koponen, M. A., Hohenester, U., Hakala, T. K. \& Toppari, J. J. Absence of mutual polariton scattering for strongly coupled surface plasmon polaritons and dye molecules with a large Stokes shift. Phys. Rev. B 88, 085425 (2013).

25. Bahsoun, H. et al. Electronic light-matter strong coupling in nanofluidic Fabry-Pérot cavities. ACS Photonics 5, 225-232 (2018).

26. Rozenman, G. G., Akulov, K., Golombek, A. \& Schwartz, T. Long-range transport of organic exciton-polaritons revealed by ultrafast microscopy. ACS Photonics 5, 105-110 (2018).

27. Coles, D. M. et al. Strong coupling between chlorosomes of photosynthetic bacteria and a confined optical cavity mode. Nat. Commun. 5, 5561 (2014).

28. Hertzog, M. et al. Voltage-controlled switching of strong light-matter interactions using liquid crystals. Chem. Eur. J. 23, 18166-18170 (2017).

29. Thomas, A. et al. Ground-state chemical reactivity under vibrational coupling to the vacuum electromagnetic field. Angew. Chem. Int. Ed. 55, 11462-11466 (2016).

30. Wang, W. et al. Interplay between strong coupling and radiative damping of excitons and surface plasmon polaritons in hybrid nanostructures. ACS Nano 8, 1056-1064 (2014).

31. Orgiu, E. et al. Conductivity in organic semiconductors hybridized with the vacuum field. Nat. Mater. 14, 1123-1129 (2015).

32. Chikkaraddy, R. et al. Single-molecule strong coupling at room temperature in plasmonic nanocavities. Nature 535, 127-130 (2016).

33. Yang, Z. et al. Recent advances in organic thermally activated delayed fluorescence materials. Chem. Soc. Rev. 46, 915-1016 (2017).

34. Kretzschmar, A., Patze, C., Schwaebel, S. T. \& Bunz, U. H. Development of thermally activated delayed fluorescence materials with shortened emissive lifetimes. J. Org. Chem. 80, 9126-9131 (2015).

35. Turro, N. J. Modern Molecular Photochemistry (University Science Books, Mill Valley, CA, 1991) 
36. Lettinga, M. P., Zuilhof, H. \& van Zandvoort, M. A. M. J. Phosphorescence and fluorescence characterization of fluorescein derivatives immobilized in various polymer matrices. Phys. Chem. Chem. Phys. 2, 3697-3707 (2000).

37. Kéna-Cohen, S. \& Forrest, S. Green polariton photoluminescence using the red-emitting phosphor PtOEP. Phys. Rev. B 76, 075202 (2007).

38. Berberan-Santos, M., Bodunov, E. \& Valeur, B. Mathematical functions for the analysis of luminescence decays with underlying distributions 1. Kohlrausch decay function (stretched exponential). Chem. Phys. 315, 171-182 (2005).

39. Deng, H., Haug, H. \& Yamamoto, Y. Exciton-polariton Bose-Einstein condensation. Rev. Mod. Phys. 82, 1489-1537 (2010).

40. Skolnick, M., Fisher, T. \& Whittaker, D. Strong coupling phenomena in quantum microcavity structures. Semicond. Sci. Technol. 13, 645 (1998).

\section{Acknowledgements}

K.B gratefully acknowledges the financial support of the Swedish Foundation for Strategic Research (ICA14-0018), the Ragnar Söderberg Foundation, the Swedish Research council (2016-03354), and the European Research council (ERC-2017-StG-757733). K.S is thankful to the Finnish Cultural Foundation and the Wenner-Gren Foundation for supporting this work. Bo Albinsson is acknowledged for providing access to spectrofluorometer and flash-photolysis apparatus.

\section{Author contributions}

K.B. conceived the idea and supervised the project; K.S and K.B designed the experiments, K.S. conducted the experiments and analyzed the results, M.H. performed the dispersion analysis and simulations. All the authors contributed to writing the manuscript.

\section{Additional information}

Supplementary Information accompanies this paper at https://doi.org/10.1038/s41467018-04736-1.

Competing interests: The authors declare no competing interests.

Reprints and permission information is available online at http://npg.nature.com/ reprintsandpermissions/

Publisher's note: Springer Nature remains neutral with regard to jurisdictional claims in published maps and institutional affiliations.

(c) (i) Open Access This article is licensed under a Creative Commons Attribution 4.0 International License, which permits use, sharing, adaptation, distribution and reproduction in any medium or format, as long as you give appropriate credit to the original author(s) and the source, provide a link to the Creative Commons license, and indicate if changes were made. The images or other third party material in this article are included in the article's Creative Commons license, unless indicated otherwise in a credit line to the material. If material is not included in the article's Creative Commons license and your intended use is not permitted by statutory regulation or exceeds the permitted use, you will need to obtain permission directly from the copyright holder. To view a copy of this license, visit http://creativecommons.org/ licenses/by/4.0/.

(c) The Author(s) 2018 